\title{
Coincidental Occurrence of Acute In-stent Thrombosis and latrogenic Vessel Perforation During a Wingspan Stent Placement: Management with a Stent In-stent Technique
}

\author{
Sun Joo Lee, MD, Hee Sup Shin, MD, Seung Hwan Lee, MD, Jun Seok Koh, MD
}

We presented a case that an acute in-stent thrombosis after the deployment of a Wingspan stent was successfully managed with a stent in-stent technique. Because vessel perforation and subarachnoid hemorrhage were iatrogenically developed during the procedure, we were unable to use the thrombolytic agents to correct the in-stent thrombosis. When a thrombotic complication following an intracranial stent placement occurs with a coincidentally hemorrhagic complication, the stent in-stent technique should be considered as a treatment option.

Key Words : Wingspan stent; In-stent thrombosis; Stent in-stent technique

Atherosclerotic intracranial arterial stenosis is one of the most common causes of stroke worldwide and is associated with a high risk of recurrent stroke. Symptomatic severe stenoses are at particularly high risk of recurrent stroke despite medical management [1]. Therefore, relative limitations of the medical management have encouraged the development of new strategies, such as endovascular treatment. Currently, endovascular therapy is increasing in popularity in the treatment of symptomatic intracranial atherosclerosis.

The Wingspan and Gateway percutaneous translumi-

All authors: Department of Neurosurgery, Kyung Hee University Hospital at Gangdong, Seoul, Korea

Received July 14, 2011;

accepted after revision September 2, 2011.

Correspondence to: Jun Seok Koh, MD, Department of Neurosurgery, Kyung Hee University Hospital at Gangdong, 892 Dongnam-ro, Sangil-dong, Gangdong-gu, Seoul 134-090, Korea.

Tel. 82.2.440.6145 Fax. 82.2.440.7171

E-mail: gjs@khnmc.or.kr

This is an Open Access article distributed under the terms of the Creative Commons Attribution Non-Commercial License (http://creativecommons.org/licenses/by-nc/3.0) which permits unrestricted non-commercial use, distribution, and reproduction in any medium, provided the original work is properly cited. nal angioplasty balloon system(Stryker Neurovascular, Fremont, CA, U.S.A.), which widens a narrowed intracranial artery, is the only system currently approved by the U.S. Food and Drug Administration for certain high-risk stroke patients. The Wingspan stent is designed to be self-expandable and more flexible to accommodate fragile brain arteries. However, in several published cohorts, the periprocedural complication rate, including stroke, hemorrhage, and vessel rupture, ranged from 4.5 to $14.7 \%$ [2-4]. In-stent thrombosis (IST) is considered as one of important complication of the Wingspan. It is commonly revealed in acute or subacute phase, and it can lead to devastating results. Therefore, prompt and effective management of IST is essential to achieve patient's cure.

We present a case that acute IST after deployment of a Wingspan stent was successfully managed with another self-expandable stent without using a glycoprotein IIb/IIIa receptor antagonist or fibrinolytic agent.

\section{CASE REPORT}

A 66-year-old female was presented to the 


\section{Acute In-stent Thrombosis During a Wingspan Stent Placement}

emergency room with sudden weakness on her left side and dysarthria while taking aspirin. She had already suffered a transient ischemic attack 3 days ago. Diffusion weighted magnetic resonance imaging (MRI) showed several small acute infarctions scattered in her right frontal cortex and basal ganglia. MR angiography revealed a severe stenosis of the right middle cerebral artery (MCA). Digital subtraction angiography (DSA) confirmed the severe long segment stenosis of the MCA and a significant decrease of flow velocity to the distal segment (Fig. 1A). We suspected that her current medical treatment would be ineffective to prevent another stroke, and thus, we planned an endovascular treatment. An endovascular procedure was performed 1 week later when her left-sided weakness was almost recovered. She received a daily oral administration of $325 \mathrm{mg}$ of aspirin and $75 \mathrm{mg}$ of clopidogrel before the procedure.

Endovascular procedure was performed under local anesthesia and with systemic heparinization. With a 6
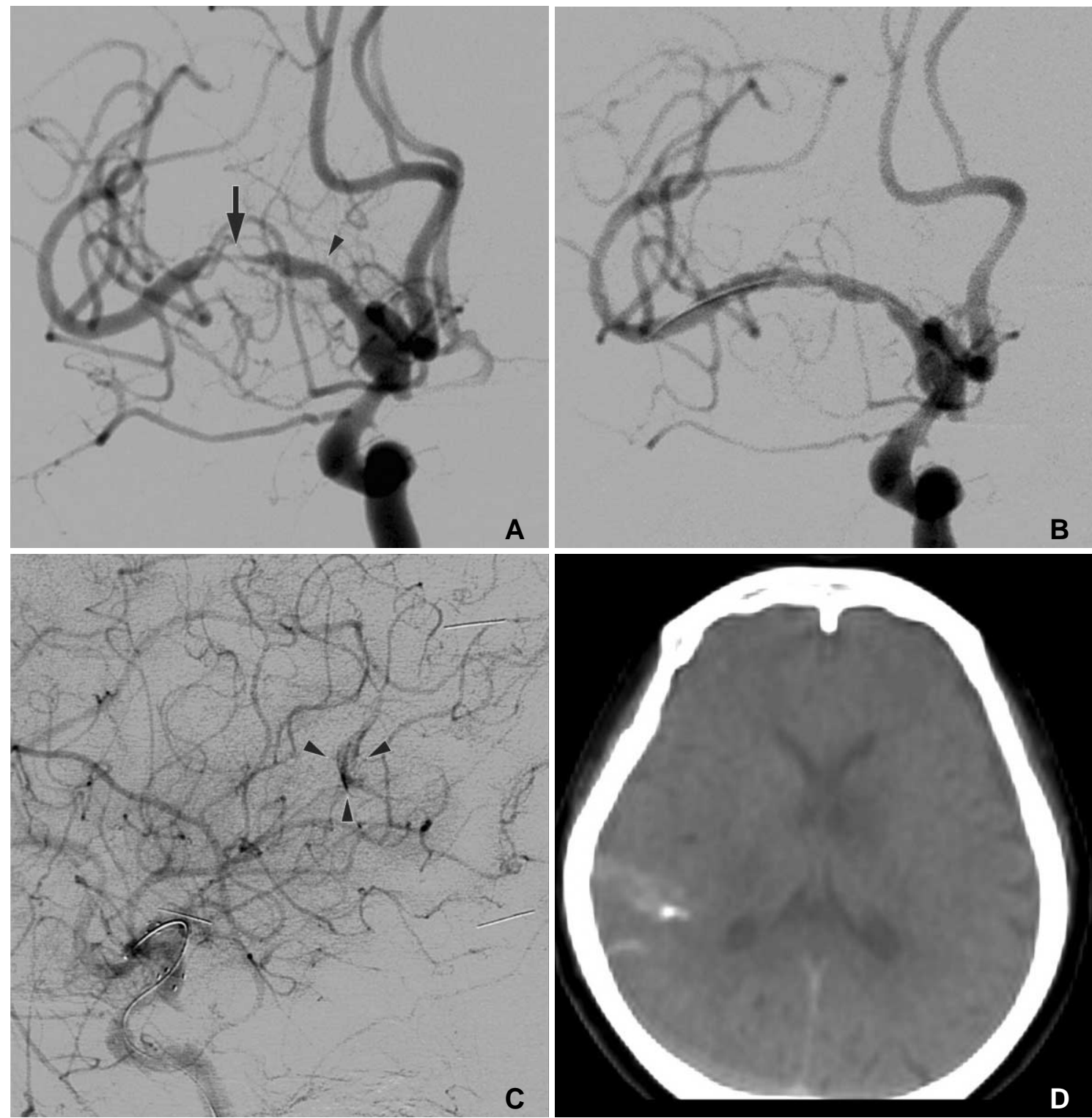

Fig. 1. A. Right internal carotid angiogram demonstrating a moderate stenosis of proximal M1 (arrowhead) and severe stenosis of distal M1 (arrow).

B. Following angioplasty and stenting with $3.5 \times 20 \mathrm{~mm}$ Wingspan system, a significant recanalization of stenotic segment with minimal residual stenosis is shown.

C. Subtle extravasation of contrast media (arrowheads) at an angular portion of the lateral sulcus is shown on the lateral right carotid angiogram.

D. Rotating flat panel CT revealing a small amount of extravasation of contrast media and subarachnoid hemorrhage at the right lateral sulcus and parietal sulci.

Continued 

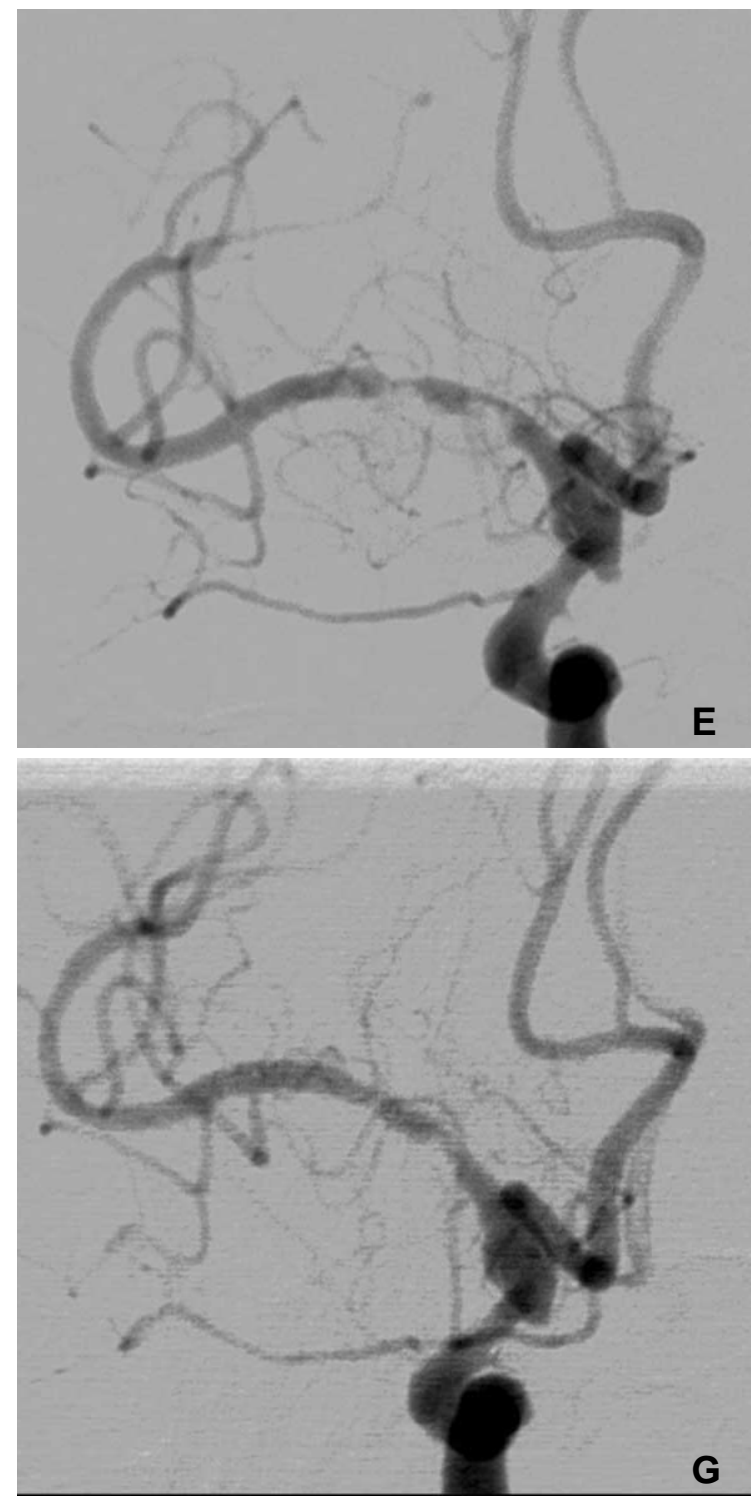

Fr guiding catheter located in the right internal carotid artery (ICA), a 010 microcatheter, and a 0.014 -inch microguidewire was navigated into the MCA. After the microcatheter was advanced across the lesion, the microwire was exchanged with a $300 \mathrm{~cm}$ exchange wire that could facilitate balloon and stent navigation. The microcatheter was removed, and a $1.5 \times 20 \mathrm{~mm}$ gateway balloon catheter was advanced over the exchange wire. The balloon was located within the stenotic segment and inflated to approximately 6 atm. On control angiogram after balloon angioplasty, stenotic MCA segment was partially recanalized. Next, the Wingspan delivery system was prepared and advanced over the exchange wire across the target lesion. During Wingspan $(3.5 \times 20 \mathrm{~mm})$ passed cavernous ICA, patient complained headache and

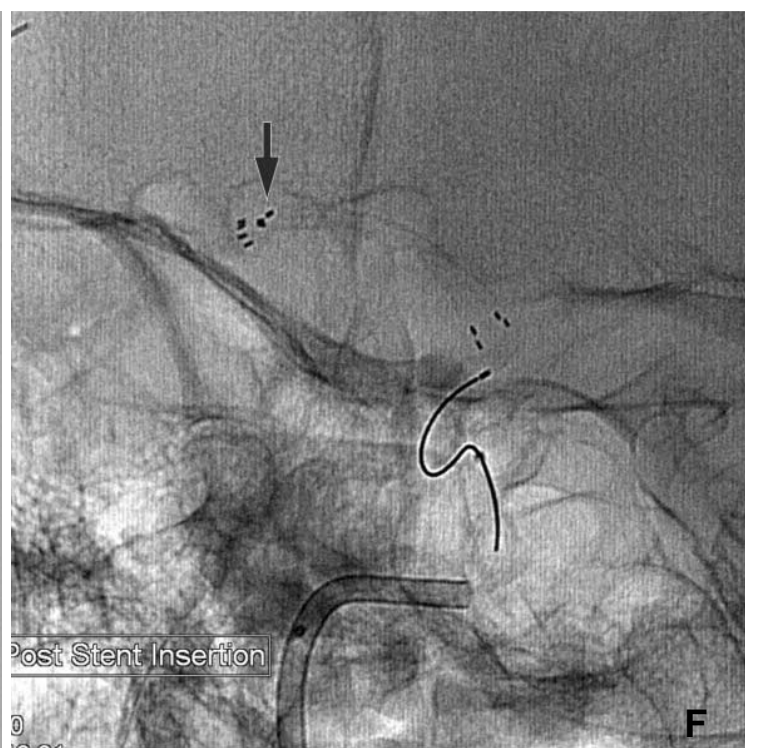

Fig. 1. E. 30-minute delayed angiogram demonstrating multifocal acute in-stent thrombosis, which compromises the flow to distal branches of right middle cerebral artery (MCA).

F. Solitaire stent $(4 \times 20 \mathrm{~mm})$ is deployed within the Wingspan stent to manage the acute in-stent thrombosis. Arrow denotes distal markers of Solitaire stent.

G. 30-minute delayed angiogram after 2nd stent deployment, thrombosis within the Wingspan stent is resolved, and MCA is reopened.

nausea, and systolic blood pressure was elevated from normal range to approximately $160 \mathrm{mmHg}$. Immediately, a stent was deployed at the stenotic segment and a control angiogram was achieved. Although the stenotic segment was recanalized and the distal flow restored to normal velocity (Fig. 1B), a tiny irregularity of posterior parietal branch of right MCA and extravasation of contrast media suggested that dissections were revealed (Fig. 1C). Rotational flat panel CT was performed immediately and showed a small amount of extravasation of contrast media within the subarachnoid space along the right lateral sulcus (Fig. 1D). We dropped the blood pressure under normal range and reversed heparin with protamine sulfate. On the angiogram performed 10 minutes later, a small filling defect appeared within the stenosis, which was 
suspected as IST. Thirty minutes later, growing thrombus reduced the flow velocity of MCA (Fig. 1E), and neurologic examination showed left-sided hemiplegia. Because of a subarachnoid hemorrhage caused by an iatrogenic vessel injury, IIb/IIIa receptor antagonist or fibrinolytic agent should not be administrated for thrombolysis. To manage the IST, we considered a mechanical recanalization using a stent in-stent technique. Rebar 18" microcatheter (ev3, Irvine, CA, U.S.A.) was passed the Wingspan stent, and a Solitaire stent $(4 \times 20 \mathrm{~mm}$, ev3, Irvine, CA, U.S.A.) was deployed to cover the Wingspan stent (Fig. 1F). Postprocedural angiogram revealed that the thrombosed segment was partially recanalized. IST was completely resolved on DSA obtained at 30 and 60 minutes after procedure (Fig. 1G), and her neurologic deficit had almost fully recovered. Brain CT at immediate and 24 hours after procedure did not reveal any new low attenuation or progression of subarachnoid hemorrhage. Seven days later, she was discharged without developing neurological sequelae.

\section{DISCUSSION}

In-stent restenosis occurs in approximately $30 \%$ of patients after delivery of the Wingspan stent, although many of these incidents of re-stenosis remain asymptomatic [5]. There has been little literature on acute IST following a Wingspan stent placement. Although the actual rate of periprocedural IST of intracranial stent is not obvious, the most recent report demonstrated that the rate of acute or subacute IST after intracranial stent ranged from $10 \%$ to $14.6 \%[4,6]$. Contrary to chronic in-stent stenosis, acute IST can result in permanent disability of the patient.

The etiology of acute IST is not fully understood. It is suspected to be associated with aspirin or clopidogrel resistance. Asprin and clopidogrel resistance occur in approximately $17 \%$ of patients undergoing coronary stent [7]. Aspirin and clopidogrel resistance may be the actual causes of IST during the periprocedural period, but it is likely there are other factors that have not been elucidated yet. It should be noted that the incidence of IST in the Wingspan and Gateway system is more frequent than that in the stent for aneurysm neck remodeling. The reason can be explained that the underlying disease itself is different from cerebral aneurysms and may be more prone to acute thrombus formation. A balloon angioplasty before the Wingspan placement may result in an endothelial injury or the exposure of atheromatous plaque material, promoting an in-stent thrombus formation [4].

The present case showed that the secondary selfexpandable stent had resolved acute IST without a new thrombus formation within the stent in spite of the nonadministration of fibrinolytic agents. The second stent would open circumferentially to displace the thrombus, immediately reestablishing a channel of flow within the occluded vessel. Once antegrade flow was restored, thrombus dissolution via endogenous thrombolysis would follow stent deployment. Therefore, we suspected that the etiology of acute IST in our case might be not associated with aspirin or clopidogel resistance but with endothelial injury. Stent in-stent would cover the exposure plaque material from the coagulation system, protecting re-growth of the thrombus within stent. Unfortunately, we were unable to confirm drug resistance using blood assay, such as VerifyNow ${ }^{\circledR}$ P2Y12 Test.

The management described for the IST includes a systemic or local application of IIb/IIIa receptor antagonist or a recombinant tissue-type plasminogen activator with/without balloon angioplasty $[4,6,8]$. Although these thrombolytic agents are effective solutions for acute thrombolysis, they cannot be used and the mechanical technique should be considered for the recanalization of thrombotic occlusion of artery when there is a recent hemorrhage such as the present case. We have found several reports regarding the use of stent-in stent technique for recanalization of the late in-stent restenosis following the Wingspan placement $[5,8,9]$. However, to the best of our knowledge, this present case is the first case that has recanalized acute IST using a stent in-stent technique and without using the thrombolytics.

Iatrogenic vascular perforation is a complication of major concern that can result in fatal intracranial hemorrhages during endovascular neurointervention. The rate of vascular perforation related to neurointerventions within the largest series was $1.1 \%$ [10]. When vascular perforation is detected during a procedure, the treatment should immediately begin with a reversal of anticoagulant, lowering the blood pressure and/or temporary or permanent closure of the ruptured vessel.

As in this case, when an IST following a Wingspan stent placement for intracranial stenosis occurs with a coincidentally hemorrhagic complication, the deployment of another stent in-stent should be considered as a treatment option.

\section{References}

1. Chimowitz MI, Kokkinos J, Strong J, Brown MB, Levine SR, 


\section{Sun Joo Lee, et al.}

Silliman S, et al. The warfarin-aspirin symptomatic intracranial disease study. Neurology 1995;45:1488-1493

2. Bose A, Hartmann M, Henkes H, Liu HM, Teng MM, Szikora I, et al. A novel, self-expanding, nitinol stent in medically refractory intracranial atherosclerotic stenoses: the Wingspan study. Stroke 2007;38:1531-1537

3. Fiorella D, Levy EI, Turk AS, Albuquerque FC, Niemann DB, Aagaard-Kienitz B, et al. US multicenter experience with the Wingspan stent system for the treatment of intracranial atheromatous disease: periprocedural results. Stroke 2007;38:881-887

4. Lawson MF, Fautheree GL, Waters MF, Decker DA, Mocco JD, Hoh BL. Acute intraprocedural thrombus formation during Wingspan intracranial stent placement for intracranial atherosclerotic disease. Neurosurgery 2010;67:ons166-170

5. Fiorella DJ, Levy EI, Turk AS, Albuquerque FC, Pride GL, Jr., Woo HH, et al. Target lesion revascularization after Wingspan: assessment of safety and durability. Stroke 2009;40:106-110

6. Riedel CH, Tietke M, Alfke K, Stingele R, Jansen O. Subacute stent thrombosis in intracranial stenting. Stroke 2009;40:13101314

7. Grossmann R, Sokolova O, Schnurr A, Bonz A, Porsche C, Obergfell A, et al. Variable extent of clopidogrel responsiveness in patients after coronary stenting. Thromb Haemost 2004;92: 1201-1206

8. Levy EI, Turk AS, Albuquerque FC, Niemann DB, AagaardKienitz B, Pride L, et al. Wingspan in-stent restenosis and thrombosis: incidence, clinical presentation, and management. Neurosurgery 2007;61:644-650

9. Le TM, Gaughen JR, Jensen ME, Evans AJ. Symptomatic Wingspan stent stenosis and occlusion: stent-in-stent rescue. $J$ Neurointerv Surg 2010;2:348-350

10. Halbach VV, Higashida RT, Dowd CF, Barnwell SL, Hieshima GB. Management of vascular perforations that occur during neurointerventional procedures. AJNR Am J Neuroradiol 1991;12:319-327 